\title{
Julia Fischer
}

\section{Evolution der Kommunikation}

Im Zentrum des Forschungsinteresses der Abteilung Kognitive Ethologie steht die Evolution der Kommunikation. Ausgehend von der Analyse kommunikativer Prozesse als einer Interaktion von Sender und Empfänger ergeben sich zwei komplementäre Forschungsstränge: erstens die Aufklärung der selektiven Drücke und evolutionären Beschränkungen, die auf das Signalverhalten des Senders wirken, und zweitens die Erforschung der kognitiven Fähigkeiten und der Verhaltenssteuerung, die der Verarbeitung von und den Reaktionen auf Signale(n) zugrunde liegen. Um die Evolution der Struktur von Signalen und des Signalverhaltens besser $\mathrm{zu}$ verstehen, nutzen wir vorwiegend Paviane (Gattung Papio) sowie Grüne Meerkatzen (Gattung Chlorocebus) als Modelle. Um die Grundlagen der Variation von Sozial-

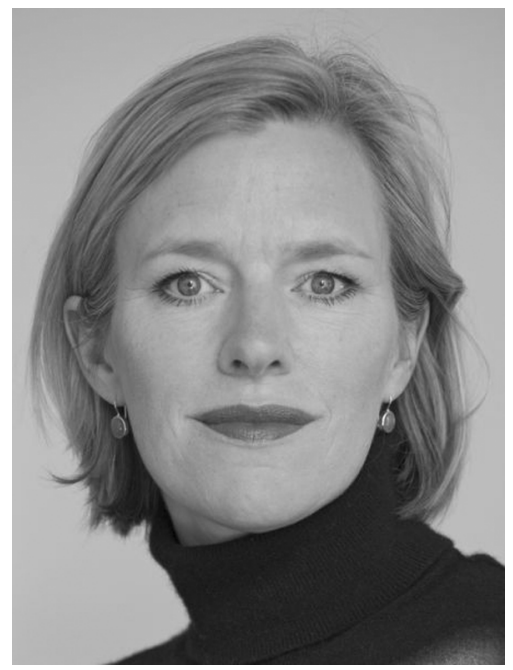

Prof. Dr. Julia Fischer, Abteilung Kognitive Ethologie, Deutsches Primatenzentrum, Göttingen und Georg-August-Universität Göttingen verhalten und Kommunikation zu quantifizieren, arbeiten wir derzeit vornehmlich an der Beschreibung und Analyse des sozialen Systems einer bislang wenig beachteten Pavianart, den Guineapavianen (Papio papio).

Ein Schwerpunkt unserer Tätigkeit in den vergangenen Jahren lag somit im Aufbau der Feldstation CRP Simenti im Senegal, die wir 2007 gründeten. Seit 2010 ist es möglich, systematische Verhaltensdaten der dort lebenden Guineapaviane von etwa 180 habituierten Individuen zu erheben. Über Guineapaviane lagen zuvor nur wenige und sich teilweise widersprechende Angaben vor. Unsere Studie wird damit einen wesentlichen Teil dazu beitragen, die Diversität in den Sozialsystemen von Pavianen zu erfassen und zu analysieren. Unsere Untersuchungen der Guineapaviane im Senegal ergaben soweit, dass sich ihre soziale Organisation und ihr Paarungssystem erheblich von Savannen- wie auch Mantelpavianen unterscheiden, und die soziale Organisation von Pavianen eine größere Diversität aufweist als bislang angenommen. Bei den Guineapavianen zeichnet sich ein so genanntes „Multi-Level-System“ ab, bei dem es verschiedene Ebenen gibt, die sich zu größeren Verbänden zusammenschließen (Patzelt et al. 2011). Auf der untersten Ebene befinden sich „Ein-Mann-Gruppen“ von männlichen 
Tieren, die exklusive Beziehungen zu einer geringen Anzahl von Weibchen unterhalten. Diese Ein-Mann-Gruppen, denen zuweilen „sekundäre“ männliche Tiere angegliedert sind, schließen sich zu sogenannten „Parties“ zusammen, die mit anderen Parties sogenannte Gangs bilden (Patzelt et al. 2014), die wiederum Teil einer größeren Community sind. Insgesamt zeigen die Tiere eine hohe Toleranz zwischen Gruppen und es gibt starke Beziehungen zwischen Männchen, die bei anderer Paviantaxa eher unüblich sind. Da Paviane auch als ein wichtiges Modell für das Verständnis der Evolution des Menschen gelten, sind diese Erkenntnisse für eine breite wissenschaftliche Gemeinde von Interesse (Patzelt et al. 2014). Zudem konnten wir zeigen, dass sich ihre Lautgebung strukturell kaum von der anderer Paviane unterscheidet, wohl aber bedeutsame Unterschiede im Einsatz der Laute zu beobachten sind (Maciej et al. 2013b). Playbackexperimente ergaben, dass männliche Guineapaviane ihre soziale Aufmerksamkeit vor allem auf Gangmitglieder richten, Nachbarn und Fremde dagegen weitgehend ignorieren, was im Einklang mit der hohen Toleranz zwischen Gruppen steht (Maciej et al. 2013a).

Zudem konnten wir in einem direkten Vergleich nachweisen, dass männliche Guineapaviane sehr viel geringere Aggressionsraten untereinander als auch gegenüber weiblichen Tieren aufweisen als Bärenpaviane. Daher eignen sich die Taxa möglicherweise, um die genetischen Grundlagen aggressiven Verhaltens zu beleuchten. Wir untersuchten die Gattung bezüglich möglicher Unterschiede in Genen, die mit dem serotonergen System der Tiere assoziiert sind. Sogenannte Polymorphismen in der Promotorregion dieser Gene, die die Konzentration des Botenstoffs Serotonin beeinflussen, wurden beim Menschen mit Variation in der Bereitschaft zu aggressivem Verhalten assoziiert (Kalbitzer et al. 2013). Allerdings fanden wir nur in einem der beiden untersuchten Genabschnitte tatsächliche Unterschiede zwischen verschiedenen Populationen (Dissertation U. Kalbitzer 2014), was entweder nahelegt, dass die betreffenden Gene nur mittelbar eine Rolle spielen, oder aber die Unterschiede eher auf der Ebene der Genregulation zu suchen sind.

Ein weiterer Schwerpunkt ist die Analyse von Lauten bei Vertretern der Gattung Chlorocebus. Diese zu den Meerkatzen gehörenden Affen spielen seit den 1980er Jahren eine herausragende Rolle in der Erforschung der Bedeutung von Lautmustern und möglichen Einsichten in den Ursprung der menschlichen Sprache. Akustische Analysen der Lautgebung von Grünen Meerkatzen im Senegal, sowie anderen Vertretern dieser Gattung werden derzeit abgeschlossen. Die vorliegenden Ergebnisse weisen darauf hin, dass eines der klassischen Modelle im Verständnis der vokalen Kommunikation von Affen und der Evolution der menschlichen Sprache einer gründlichen Revision bedarf. Zudem haben wir uns an der theoretischen Debatte über die Bedeutung von Lautmustern von Affen 
beteiligt (Wheeler \& Fischer, 2012; Fischer 2013). Ergänzt werden diese Arbeiten durch Studien zur Aufklärung des Zusammenhangs von phylogenetischen Verwandtschaftsverhältnissen und akustischer Kommunikation (Price et al. 2014). Eine entsprechende Untersuchung im Genus Papio soll demnächst abgeschlossen werden. Diese Ergebnisse untermauern die Bedeutung der angeborenen Komponente der Lautgebung nichtmenschlicher Primaten. Darüber hinaus sind wir an einer Reihe von Studien beteiligt, in der es um die Aufklärung der Funktion einzelner Gene bei der Kommunikation und des Sozialverhaltens geht (Übersicht in Fischer and Hammerschmidt 2011). Diese Studien, ebenso wie eine kürzlich erschienene Studie zur Rolle des vokalen Lernens (Hammerschmidt et al. 2012), können derzeit in dieser Form nur an Mausmodellen durchgeführt werden.

Um ein vertieftes Verständnis der kognitiven Grundlagen der Verarbeitung von Reizen und den Entscheidungsprozessen von Altweltaffen zu entwickeln, führen wir zusätzlich kognitive Tests unter kontrollierten Bedingungen an einer Gruppe von Javaneraffen am DPZ durch. Die Studien zu den kognitiven Leistungen von Javaneraffen (und anfangs auch Anubispavianen) ergaben, dass sich ihre Fähigkeiten in den betreffenden Tests nicht substantiell von den Fähigkeiten von Menschenaffen unterscheiden (Schmitt et al. 2012). Diese auf der sogenannten „Primate Cognition Test Battery“ beruhenden Befunde haben eine hohe Anzahl von Anschlussfragen aufgeworfen, die insbesondere den Einfluss spezifischer Testbedingungen auf die Leistung der Tiere betreffen (Schmitt \& Fischer 2011; Schloegl et al. 2013; Schmitt et al. 2014). So konnten wir nachweisen, dass, anders als erwartet, nicht die Stimulusqualität, sondern vor allem die Kontingenzen in der Belohnung der Tiere ihre Performanz beeinflusst (Schmitt \& Fischer 2011). Inzwischen erforschen wir im Rahmen einer Forschergruppe an der Universität Köln, ob die kognitive Leistung der Tiere in bestimmten Tests durch soziale Vergleichsprozesse moduliert werden kann. Des Weiteren überprüfen wir den Einfluss des Alterns auf Sozialverhalten und Kognition bei Berberaffen. Wir kombinieren dazu Beobachtungen des Sozialverhaltens mit kognitiven Tests bezüglich der allgemeinen Motivation und der sozialen Selektivität der Tiere (Laura Almeling Dissertation).

In der Gesamtschau sollen diese Studien dazu beitragen, erstens den Zusammenhang zwischen Sozialverhalten, Kommunikation und Intelligenz genauer zu beleuchten. Zum Zweiten zielen wir auf ein besseres Verständnis sowohl der proximaten wie auch der ultimaten Ursachen des Sozialverhaltens von Primaten ab. 


\section{Literatur}

Fischer, J. 2013. Information, Inference and Meaning in Primate Vocal Behaviour. In: Animal Communication Theory: Information and Influence, (Ed. by U. Stegmann), pp. 297-317. Cambridge: Cambridge University Press.

Fischer, J. \& Hammerschmidt, K. 2011. Ultrasonic vocalizations in mouse models for speech and socio-cognitive disorders: insights into the evolution of vocal communication. Genes, Brain and Behavior, 10, 17-27.

Hammerschmidt, K., Reisinger, E., Westekämper, K., Ehrenreich, H., Strenzke, N. \& Fischer, J. 2012. Mice do not require auditory input for the normal development of their ultrasonic vocalizations. BMC Neuroscience, 13, 40.

Kalbitzer, J., Kalbitzer, U., Knudsen, G. M., Cumming, P. \& Heinz, A. 2013. How the cerebral serotonin homeostasis predicts environmental changes: a model to explain seasonal changes of brain 5-HTT as intermediate phenotype of the 5-HTTLPR. Psychopharmacology, 230, 333-343.

Maciej, P., Patzelt, A., Ndao, I., Hammerschmidt, K. \& Fischer, J. 2013a. Social monitoring in a multilevel society: a playback study with male Guinea baboons. Behavioral Ecology and Sociobiology, 67, 61-68.

Maciej, P., Ndao, I., Hammerschmidt, K. \& Fischer, J. 2013b. Vocal communication in a complex multi-level society: constrained acoustic structure and flexible call usage in Guinea baboons. Frontiers in Zoology, 10, 58.

Patzelt, A., Zinner, D., Fickenscher, G., Diedhou, S., Camara, B., Stahl, D. \& Fischer, J. 2011. Group composition of Guinea baboons (Papio papio) at a water place suggests a fluid fission-fusion social organisation. International Journal of Primatology, 32, 652-668.

Patzelt, A., Kopp, G. H., Ndao, I., Kalbitzer, U., Zinner, D. \& Fischer, J. 2014. Male tolerance and male-male bonds in a multilevel primate society. Proceedings of the National Academy of Sciences, in press.

Price, T., Ndiaye, O. \& Fischer, J. 2014. Limited geographic variation in the acoustic structure of and responses to adult male alarm barks of African green monkeys. Behavioural Ecology and Sociobiology, 68, 815-825.

Schloegl, C., Waldmann, M. R. \& Fischer, J. 2013. Understanding of and reasoning about object-object relationships in long-tailed macaques? Animal Cognition, 16, 493-507.

Schmitt, V. \& Fischer, J. 2011. Representational format determines numerical competence in monkeys. Nature Communications, 2, 257.

Schmitt, V., Pankau, B. \& Fischer, J. 2012. Old World Monkeys Compare to Apes in the Primate Cognition Test Battery. PLoS One, 7, e32024.

Schmitt, V., Schloegl, C. \& Fischer, J. 2014. Seeing the Experimenter Influences the Response to Pointing Cues in Long-Tailed Macaques. PLOS ONE, 9, e91348.

Wheeler, B. C. \& Fischer, J. The blurred boundaries of functional reference: a response to Scarantino \& Clay. Animal Behaviour,

Wheeler, B. C. \& Fischer, J. 2012. Functionally referential signals: a promising paradigm whose time has passed. Evolutionary Anthropology, 21, 195-205. 